\title{
Suppressed smooth muscle proliferation and inflammatory cell invasion after arterial injury in elafin-overexpressing mice
}

\author{
Syed H.E. Zaidi, ${ }^{1,2}$ Xiao-Mang You, ${ }^{3,4}$ Sorana Ciura, ${ }^{1}$ Stacey O’Blenes, ${ }^{1,5}$ \\ Mansoor Husain, ${ }^{3,4}$ and Marlene Rabinovitch $1,2,4,6$ \\ ${ }^{1}$ Program in Cardiovascular Research, The Hospital for Sick Children, \\ ${ }^{2}$ Department of Laboratory Medicine and Pathobiology, \\ ${ }^{3}$ Center for Cardiovascular Research, University Health Network, \\ ${ }^{4}$ Department of Medicine, \\ ${ }^{5}$ Department of Surgery, and \\ ${ }^{6}$ Department of Pediatrics, University of Toronto, Toronto, Ontario, Canada
}

Address correspondence to: Marlene Rabinovitch, Division of Cardiovascular Research, Hospital for Sick Children, 555 University Avenue, Toronto, Ontario, Canada M5G 1X8. Phone: (416) 813-5918; Fax: (416) 813-7480;

E-mail: mr@sickkids.on.ca.

Received for publication December 14, 1999, and accepted in revised form May 9, 2000.

\begin{abstract}
Elastases degrade the extracellular matrix, releasing growth factors and chemotactic peptides, inducing glycoproteins such as tenascin, and thereby promoting vascular cell proliferation and migration. Administration of serine elastase inhibitors reduces experimentally induced vascular disease. The ability to mount an intrinsic anti-elastase response may, therefore, protect against inti$\mathrm{mal} / \mathrm{medial}$ thickening after vascular injury. To investigate this, we showed that wire-induced endothelial denudation of the carotid artery is associated with transient elevation in elastase activity and confirmed that this is abolished in transgenic mice overexpressing the serine elastase inhibitor, elafin, targeted to the cardiovascular system. Ten days after injury, nontransgenic littermates show vessel enlargement, intimal thickening, increased medial area and cellularity, and 2 -fold increase in tenascin. Injured vessels in transgenic mice become enlarged but are otherwise similar to sham-operated controls. Injury-induced vessel wall thickening, which is observed only in nontransgenic mice, is related to foci of neutrophils and macrophages, in addition to smooth muscle cells that fail to stain for $\alpha$-actin and are likely dedifferentiated. Our study therefore suggests that a major determinant of the vascular response to injury is the early transient induction of serine elastase activity, which leads to cellular proliferation and inflammatory cell migration.
\end{abstract}

J. Clin. Invest. 105:1687-1695 (2000).

\section{Introduction}

Heightened serine elastase activity has been documented in several experimentally induced cardiovascular diseases (1-3). Serine elastases can stimulate remodeling of the heart and blood vessels by direct effects and by their ability to activate a proteolytic cascade involving other enzymes such as matrix metalloproteinases (4-6). In response to elastase-mediated degradation of the extracellular matrix, growth factors $(7,8)$ and cytokines $(9)$ are released and there is induction of glycoproteins such as tenascin and fibronectin, which enhance cell proliferation and migration, respectively $(10,11)$. We have previously shown that administration of highly selective serine elastase inhibitors reduce pulmonary vascular disease in rats (1), postcardiac transplant coronary artery disease and myocardial rejection in rabbits (2), and myocarditis in mice $(3,12)$. In this study, we evaluate the potential modulating role of endogenously expressed elastase inhibitors by comparing intimal and medial thickening after wire-induced carotid arte- rial injury (13) in transgenic mice in which the serine elastase inhibitor, elafin, was overexpressed (12) and in nontransgenic littermates.

Elafin has been shown to selectively inhibit leukocyte elastase, pancreatic elastase, and proteinase 3, but not other serine proteinases such as trypsin, chymotrypsin, plasmin, and cathepsin G (14). Elafin cDNA encodes for a $12-\mathrm{kDa}$ protein that contains a signal peptide, a transglutaminase substrate domain, and the elafin inhibitory domain (14). The elafin signal peptide targets the protein to the cell membrane where it is cleaved, and the precursor is secreted and either cross-linked to extracellular matrix proteins via the transglutaminase domain or cleaved to produce the $6-\mathrm{kDa}$ mature elafin inhibitory protein (14). In the elafin-transgenic mice, the murine preproendothelin promoter was used to target overexpression of the human elafin transgene to the cardiovascular system. To distinguish human elafin from endogenous mouse elafin, an eight amino acid-long FLAG sequence was incorporated into the $\mathrm{COOH}$-terminus 
of human elafin. We established that this FLAGtagged human elafin was a functionally active elastase inhibitor in the transgenic mice and that they did not exhibit phenotypic abnormalities related to morphology or elastin content of heart, blood vessels, and other tissues. We did, however, show that these mice were protected from myocardial damage after encephalomyocarditis virus infection (12). This is consistent with previous studies in mice in which a protective effect of elastase inhibitors on the sequelae of viral myocarditis was documented (3).

In the present study, we show that carotid arterial injury is characterized by an early transient increase in serine elastase activity that was completely suppressed in elafin-overexpressing transgenic mice. Ten days after the injury, severe intimal and medial thickening, associated with enhanced cellularity and tenascin expression, were only apparent in nontransgenic littermates relative to sham-operated controls. The increase in cellularity in the nontransgenic mice was attributed to foci of inflammatory (Ly-6G and Mac-3 positive) cell infiltration as well as $\alpha$-actin negative cells, which are likely smooth muscle cells (SMCs) that had proliferated, migrated, and dedifferentiated. It therefore appears that intrinsic inhibition of elastase activity in the vessel wall can maintain SMC phenotype and reduce inflammatory cell migration by preserving the integrity of the matrix.

\section{Methods}

Genotyping. A detailed description of the creation of the B6/SJL-CD1 transgenic mouse in which the mouse preproendothelin promoter was used to target overexpression of FLAG-tagged human elafin to the cardiovascular system is provided elsewhere (12). All transgenic mice and nontransgenic littermates used in the present study were genotyped by dot blot hybridization of tail genomic DNA $(5-8 \mu \mathrm{g})$ to radiolabeled human elafin cDNA as described previously (12). We identified nine transgenic and six nontransgenic male mice from three different litters used in this study. Although this resulted in differences in the number of animals in each group, it also ensured that mice of matched genetic backgrounds and age were being compared.

Carotid injury. Carotid arterial injury was performed in all 15 transgenic mice and nontransgenic littermates already described here and in $25 \mathrm{CD}-1$ mice for initial characterization of the model at 8-10 weeks of age, after a protocol described previously (13). Briefly, the mice were anesthetized with an intraperitoneal injection of ketamine $100 \mathrm{mg} / \mathrm{kg}$ (MTC Pharmaceuticals, Cambridge, Ontario, Canada) and xylazine $10 \mathrm{mg} / \mathrm{kg}$ (Bayer Inc., Etobicoke, Ontario, Canada). Using a dissecting microscope (MZ6; Leica, Heerbrugg, Switzerland), the left carotid artery was exposed on the ventral side of the neck via a midline incision. The bifurcation of the carotid artery was located and two ligatures were placed around the external carotid artery, which was then tied off with the distal ligature. After temporarily occluding the internal carotid artery with a vascular clamp, a small incision was made between the two ligatures placed around the external carotid artery to introduce the endothelial denudation device, a curved flexible copper wire $(0.30 \mathrm{~mm}$ diameter). Under rotation, the wire was passed along the main carotid artery four times. The wire was removed, and the external carotid artery was tied off proximal to the incision hole. The clamp was removed and the pulse of the carotid artery was rechecked. The skin incision was closed with a single suture. Animals were allowed to recover and carotid arteries were harvested at various time points as described in Results. Carotid arteries were fixed in 4\% formalin for 30 minutes before embedding and freezing in Tissue-Tek OCT media (Miles Inc., Elkhart, Indiana, USA). Frozen sections were stained with Movat's pentachrome or hematoxylin and eosin (H\&E), and immunohistochemistry was performed using various antibodies as described in detail later here. Injured arteries from two CD1 mice, harvested at 6 hours, were also examined for inflammatory cells by light microscopy using H\&E-stained cross sections.

Assay for elastase activity. Elastase activity was assayed in sham and injured carotid arteries of CD-1 mice at 6,24 , and 48 hours and at 4 and 7 days to establish a time course. Comparisons were then made between transgenic mice and nontransgenic littermates at 6 hours after injury. Because of the limitation in size of the vessels, it was necessary to pool three or four carotid arteries for each determination as indicated in the figure legends. The elastase assay has been described in detail previously (3). Briefly, carotid arteries were harvested using the same landmarks, rinsed in PBS, and homogenized in $0.9 \% \mathrm{NaCl}$. Homogenates were then centrifuged, and pellets were extracted with $0.5 \mathrm{M}$ sodium acetate $(\mathrm{pH} 4.0)$ overnight at $4^{\circ} \mathrm{C}$. After centrifugation, supernatants from these extracts were dialyzed against water, lyophilized, and resuspended in $60 \%$ saturated ammonium sulfate. Proteins were allowed to precipitate overnight at $4^{\circ} \mathrm{C}$, and these were resuspended in 50 $\mu \mathrm{L}$ assay buffer (50 mM Tris- $\mathrm{Cl}$ [pH 8.0], $150 \mathrm{mM}$ $\mathrm{NaCl}, 10 \mathrm{mM} \mathrm{CaCl}_{2}$, and $0.02 \%$ Brij). $\mathrm{N}$-Methylamine ( $2 \mathrm{mM})$ was added to all the solutions during sample preparation to inhibit potential $\alpha 2$-macroglobulin interference with elastase activity. The elastase assay was performed using a fluorescent substrate AFC091 (40 $\mu \mathrm{M}$; Enzyme Systems, Livermore, California, USA) in $1 \mathrm{~mL}$ assay buffer, and substrate cleavage was monitored over a period of 10 minutes. The amount of elastase activity detected was calibrated against a standard curve using human leukocyte elastase (specific activity 800-900 U/mg; Elastin Products, St. Louis, Missouri, USA). To determine whether the activity monitored would be inhibited by elafin, we added recombinant human elafin $(2 \mathrm{ng} / \mathrm{mL}$ ) (a gift from J. Fitton of Zeneca, Macclesfield, United Kingdom) to the assay mixture, and a change in fluorescence was monitored over the next 10 minutes. 
Immunostaining. Frozen sections were used for immunodetection of various antigens using the Vectastain ABC kit (Vector Laboratories, Burlington, Ontario, Canada), which included relevant secondary antibodies. Rabbit anti-FLAG polyclonal antibody (1:400 dilution; Zymed Laboratories, San Francisco, California, USA) and rabbit anti-chicken tenascin polyclonal antibody (1:200) were used overnight at $4^{\circ} \mathrm{C}$. Rat anti-mouse Mac-3 and Ly-6G (15) (PharMingen, Mississauga, Ontario, Canada) were used at a concentration of $1 \mu \mathrm{g} / \mathrm{mL}$ for 1 hour at room temperature. Horseradish peroxidase-conjugated (HRP-conjugated) undiluted anti-SMC $\alpha$-actin $\mathrm{mAb}$ (DAKO Corp., Carpinteria, California, USA) was used for 1 hour at room temperature. All immune complexes were visualized by incubating in DAB (Sigma Chemical Co., St. Louis, Missouri, USA). As a negative control, anti-rabbit polyclonal antibody (Vector Laboratories) was used for tenascin and isotypic monoclonal IgG for both anti-Mac-3 and Ly6G. For actin, HRP-conjugated mouse IgG was used as a control. Sections were then counterstained with hematoxylin. Immunostaining for the proliferating cell nuclear antigen (PCNA) was also carried out on arteries 10 days after injury using a mouse $\mathrm{mAb}$ (1:100 dilution and overnight incubation at $4^{\circ} \mathrm{C}$ ) (DAKO Corp.) and mouse skin as a positive control. Secondary HRP conjugated anti-mouse IgG $2 \alpha$ antibody (1:66 dilution; Amersham Canada, Oakville, Ontario, Canada) was used to visualize immune complexes. This antibody was also used as a negative control for immunostaining.

Image analysis. Full microscopic cross sections were directly captured onto a computer and images were analyzed by the Image-Pro plus program (Media Cybernetics, Silver Springs, Maryland, USA). Immunoperoxidase staining was quantified and normalized to the total medial area. Medial area was quantified by measuring the area between the innermost and the outermost elastic laminae. The number of cells was then assessed by counting nuclei over the area of intima plus media. To determine whether there had been changes in vessel size, we measured the dimensions of the area internal to the external elastic lamina. We also determined qualitatively whether a neointima was present (deposition of cells and matrix above the internal elastic lamina), and when present, this region was quantified and expressed relative to the medial area. We also calculated a luminal area.
Statistical analysis. All data are presented as mean \pm SEM. The number of animals used for each determination is given in the figure legends. Comparisons were made with the Fisher's test. A value of $P \leq 0.05$ was considered statistically significant.

\section{Results}

Induction of elastase activity after carotid arterial injury. CD-1 mice were used to characterize wire injury of the carotid artery. We documented that the endothelium was denuded in vessels examined by light microscopy at 6 hours after injury (Figure 1a). At this time point, no inflammatory cells infiltrating or adherent to the vessel were seen by light microscopy. We also established, at this same 6-hour time point, a similar level of detectable elastase activity in two separate experiments, using pooled vessels from three or four mice. When we extended the time course of the experiment to 24 hours, 48 hours, or 4 days after injury, elastase activity was no longer detectable (Figure 1b). However, at 7 days, elastase activity was again observed in the injured arteries. Addition of recombinant elafin completely abolished the elastase activity detected at 6 hours or 7 days, indicating that the enzyme was a serine elastase. The absence of inflammatory cells adherent to or within the wall of vessels 6 hours after the injury suggested that the source of elastase activity was likely the SMCs; this is in keeping with our previous cell culture studies $(7,16,17)$. No elastase activity was detectable in the noninjured sham-operated control arteries at any time point.

To confirm expression of the elafin transgene (Figure $2 a)$, carotid arteries from elafin-transgenic and nontransgenic mice were immunostained with rabbit antiFLAG polyclonal antibodies (Figure 2b). Human elafin expression was localized to the endothelium and medial SMC layer of elafin-transgenic mice but was not apparent in nontransgenic mice.

In two independent experiments, we assessed elastase activity 6 hours after injury, comparing injured and noninjured vessels in elafin overexpressing transgenic and nontransgenic littermates (Figure 2c). Elastase activity was completely suppressed in elafin-transgenic mice, whereas in nontransgenic littermates, it was upregulated as seen in CD1 mice. No elastase activity was detected in sham-operated arteries from elafintransgenic or nontransgenic littermates.

Medial thickening. We documented a more than onefold increase in medial area in the injured arteries of

Table 1

Morphometric analysis of injured and sham operated arteries 10 days after carotid arterial injury

\begin{tabular}{lcccc}
\hline & \multicolumn{2}{c}{ Nontransgenic } & \multicolumn{2}{c}{ Elafin-transgenic } \\
Parameters & Sham & Injured & Sham & Injured \\
Area within outer elastic laminae $\left(\mu \mathrm{m}^{2}\right)$ & $82,706 \pm 23,386$ & $128,400 \pm 12,863^{\mathrm{A}}$ & $86,683 \pm 10,665$ & $123,346 \pm 7,949^{\mathrm{A}}$ \\
Luminal area $\left(\mu \mathrm{m}^{2}\right)$ & $61,362 \pm 20,341$ & $57,957 \pm 11,200$ & $59,856 \pm 9,461$ & $86,538 \pm 7,774$ \\
Medial area $\left(\mu \mathrm{m}^{2}\right)$ & $21,343 \pm 3,343$ & $48,955 \pm 6,637^{\mathrm{A}}$ & $26,827 \pm 2,928$ & $35,440 \pm 4,174^{\mathrm{A}, \mathrm{B}}$ \\
& & &
\end{tabular}


nontransgenic mice when compared with the shamoperated arteries $(P=0.0004) 10$ days after injury. In the injured arteries of elafin-transgenic mice, however, medial area was significantly less than in the nontransgenic littermates $(P=0.036)$ and was not, in fact, different from that in noninjured sham-operated arteries (Figure 3, a and b; Table 1). No differences were observed in the medial area of carotid arteries from elafin-transgenic and nontransgenic noninjured sham-operated arteries.

\section{a}
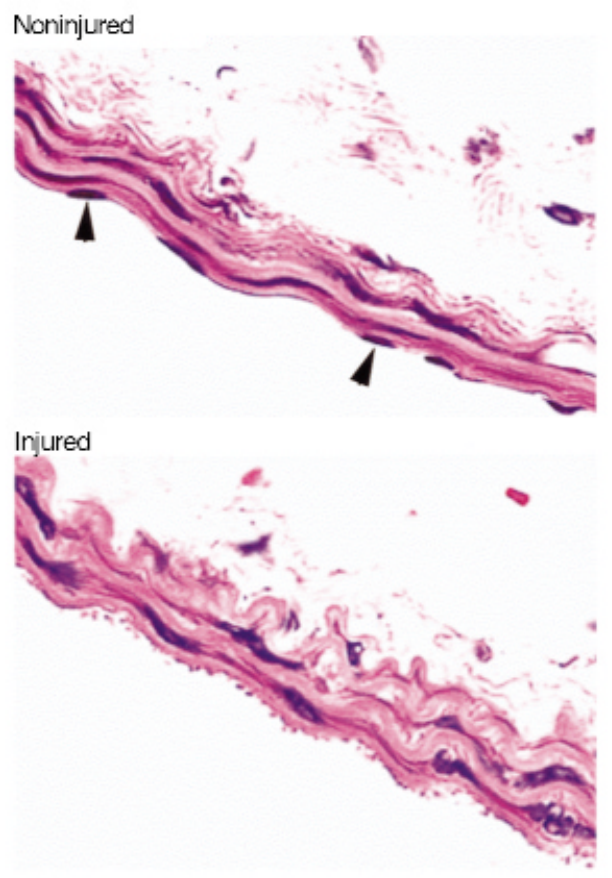

b

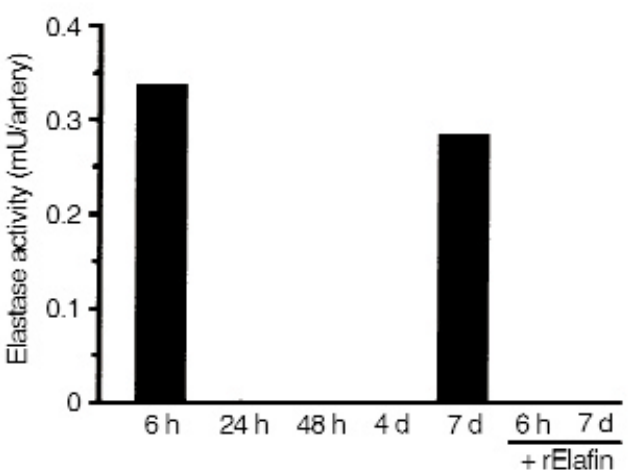

Figure 1

Carotid arterial wire injury. (a) Representative H\&E-stained sections of noninjured and injured carotid arteries (harvested 6 hours after endothelial denudation) from one of two different CD1 mice. Endothelial nuclei apparent in the noninjured vessel (arrowheads) are not seen after injury. (b) Elastase activity from pooled homogenates of three or four vessels was assayed and expressed per artery after a calculation based on a standard plot generated by using known concentrations of human leukocyte elastase. Bar at 6 hours represents an average of two separate experiments with similar values of 0.315 and $0.346 \mathrm{mU}$ per artery. Recombinant human elafin (rElafin; 2 ng) completely abolished the elastase activity observed at 6 hours and 7 days after injury.
Vessel size, assessed by planimetry using the circumference of the external elastic lamina, was similarly increased by about $40 \%$ in nontransgenic and elafin transgenic-injured compared with noninjured shamoperated mice $(P<0.05$ for both) (Table 1$)$. Neointima formation was observed in five of six nontransgenic mice in which the intima/media ratio was $0.549 \pm$ 0.254 SEM compared with one in nine elafin-transgenic mice in which the intima/media ratio was 0.341 (Figure $3 \mathrm{~d}$ ). The luminal area, although not significantly different, showed a trend toward an increase in elafin transgenic injured compared with nontransgenic-injured arteries (Table 1).

We further documented that the increase in medial area and presence of a neointima in the nontransgenicinjured carotid arteries was accompanied by an increase in cell number (Figure 3c) relative to elafin-transgenic injured arteries $(P=0.0001)$ as well as sham-operated nontransgenic vessels $(P=0.0001)$ in which values were similar. Disruption of the elastic lamina was observed in the injured arteries of four of six nontransgenic mice compared with two of 11 elafin-transgenic mice.

Extracellular matrix protein tenascin deposition. In association with pulmonary vascular disease, SMC proliferation is related to elastase-mediated induction of the extracellular matrix protein tenascin (11). We therefore assessed tenascin by quantitative immunohistochemistry as percent positive staining related to total area of intima and media in the carotid arteries 10 days after the injury. Strong tenascin expression was observed in approximately $45 \%$ of the intima plus media of injured arteries of nontransgenic mice compared with approximately $10-20 \%$ of the injured arteries in elafin-transgenic mice $(P=0.005)$, which was not significantly different from that observed in the control sham-operated arteries (Figure 4). There were no significant differences in tenascin expression comparing the sham-operated arteries of nontransgenic and elafin-transgenic mice.

Change in SMC phenotype. Previous studies have shown that in response to vascular injury, proliferating SMC may undergo changes in phenotype as judged by reduced or even absent expression of $\alpha$ smooth muscle actin (18-20). Ten days after the carotid wire injury, $\alpha$ smooth muscle actin expression in arteries of nontransgenic mice was focal and minimal to absent in the thickest part of the vessel wall. When quantified, actin immunostaining was only approximately $25 \%$ of total intima plus media area and thus considerably reduced compared with elafinoverexpressing transgenic mice in which values were approximately $60 \%(P=0.0015)$, which was not significantly different from those in sham-operated arteries (Figure 5) from nontransgenic and elafintransgenic littermates. The actin negative cells did not appear to be actively proliferating at 10 days as judged by absence of PCNA positivity (data not shown).

Although dedifferentiated SMC can be $\alpha$-actin negative, it is also possible at least some of these cells 
are inflammatory in origin, reflecting elastase-mediated transvascular trafficking. In the injured arteries of nontransgenic mice, heightened Mac-3 expression (a marker for macrophages; ref. 21) was observed in the thickest part of the media where cells were negative for $\alpha$-actin at day 10 after the injury. The increased immunostaining was $20-30 \%$ of intima plus media area, higher than in the injured arteries of elafin overexpressing transgenic mice $(P=0.006)$ where values were $\sim 10 \%$ and slightly but not significantly different from those in sham-operated arteries (Figure 6, $a$ and $b$ ).

Using a rat anti-mouse neutrophil marker, Ly-6G antigen, we also noted increased immunostaining in the arteries of nontransgenic mice 10 days after injury but the distribution was more focal (Figure 6, c and d). When quantified, in the injured arteries of nontransgenic mice, values were approximately $5-15 \%$, of intima plus media area. This was higher than the injured arteries of elafin-transgenic mice $(P=0.032)$. In fact, only two of 11 injured carotid arteries of elafin-transgenic mice showed any Ly-6G staining. No or minimal Ly-6G staining was observed in the sham-operated arteries of elafin-transgenic and nontransgenic mice. Thus, from the calculations of percentage immunoreactivity, it appears that many of the $\alpha$-actin-negative cells were inflammatory in origin.

Independent of whether elastic laminae were disrupted or intact, abundant inflammatory cells were observed in the media plus intima of the injured arteries of nontransgenic mice, and only minimal inflammatory cells were seen in the media of elafintransgenic mice. To determine whether elafin transgene expression was induced in the inflammatory cells infiltrating the media of injured arteries, frozen sections from two injured arteries of elafin transgenic mice in which neutrophils and macrophages were seen in the media, were immunostained with rabbit anti-FLAG antibodies. In both sections, immunostaining for FLAG-elafin did not colocalize with inflammatory cells. Although we cannot exclude the possibility of elafin transgene induction by the endothelin promoter in leukocytes, absence of detectable immunostaining likely indicates a low level of expression.

\section{Discussion}

In the present study, we provide direct evidence for induction of serine elastase activity as early as 6 hours after endothelial denudation of the carotid artery. We further show that this elastase activity is completely inhibited by recombinant elafin and in vivo in elafin overexpressing transgenic mice. We then address the relationship between the early induction of elastase activity and the subsequent remodeling of the vessel wall in response to injury. This model is characterized by an increase in size, thickening of the media, and the development of a neointima. We show, in elafin transgenic mice, that the injury results in an increase in vessel size but without thickening of the media or a neointima, and we attribute this to inhibition of the downstream effects of elastase activity, which include stimulation of SMC proliferation associated with induction of the glycoprotein tenascin, and transvascular migration of inflammatory cells (neutrophils and macrophages).

$\mathbf{a}$

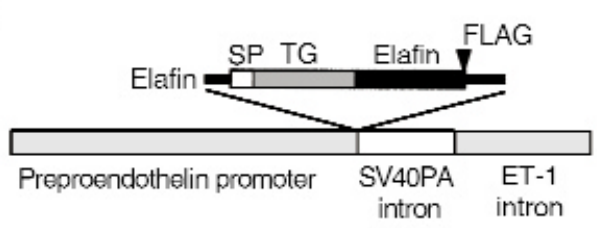

b

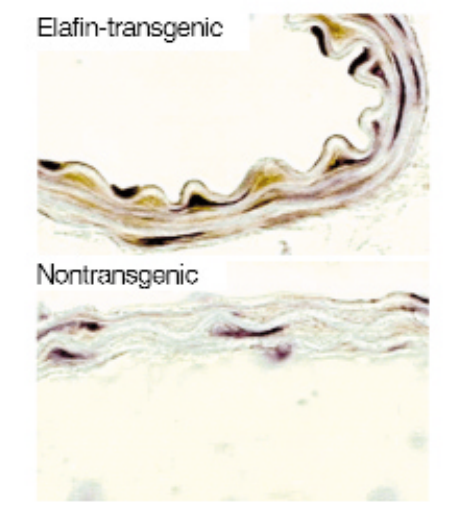

C

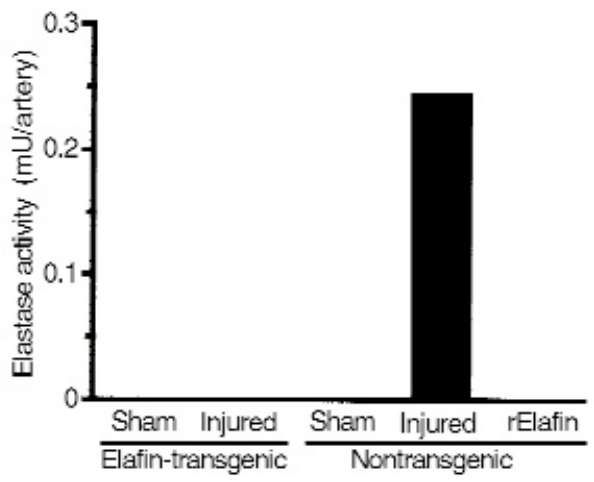

\section{Figure 2}

Elafin-overexpressing transgenic mice. (a) Diagram of the construct used to create the transgenic mice in which $\mathrm{COOH}$-terminal FLAG-tagged human elafin was cloned downstream of the mouse preproendothelin promoter. Signal peptide (SP), transglutaminase substrate (TG), and elafin inhibitory domains are denoted along the top of elafin cDNA. ET-1, endothelin-1. (b) Carotid arteries from elafin-transgenic and nontransgenic mice were immunostained with anti-FLAG antibodies before hematoxylin counterstaining. Brown immunoperoxidase staining for FLAGelafin is observed in the carotid artery from the elafin-transgenic but not the nontransgenic littermate. (c) Six hours after carotid arterial injury, sham-operated and injured arteries pooled from four different elafin-transgenic or nontransgenic mice were assayed for elastase activity. Elastase activity is expressed per artery as described in the legend to Figure 1. Recombinant human elafin (rElafin; $2 \mathrm{ng}$ ) completely abolished the elastase activity of nontransgenic-injured arteries. 
a

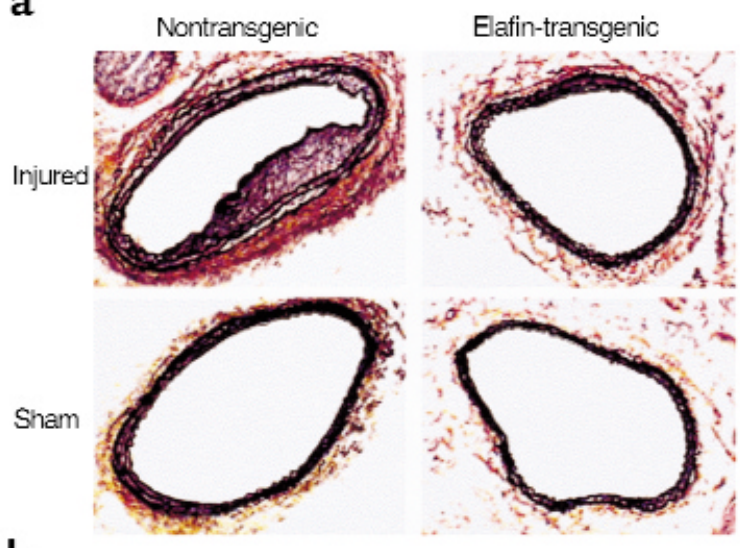

b

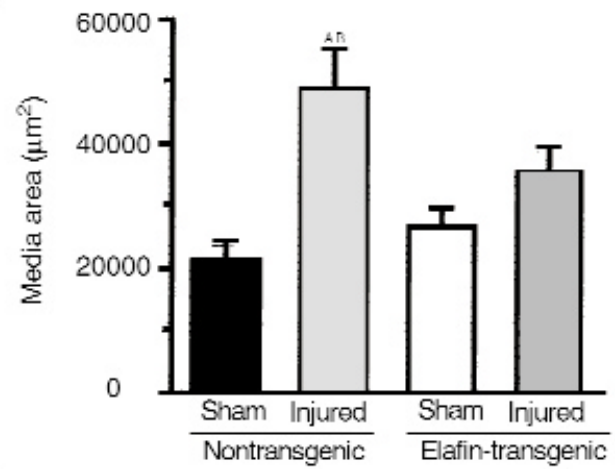

C

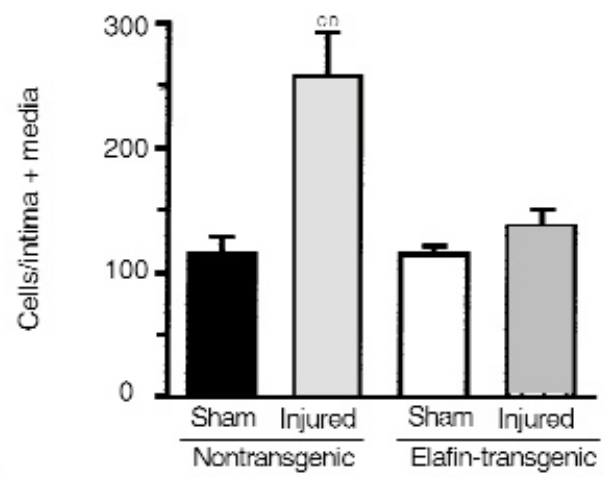

d

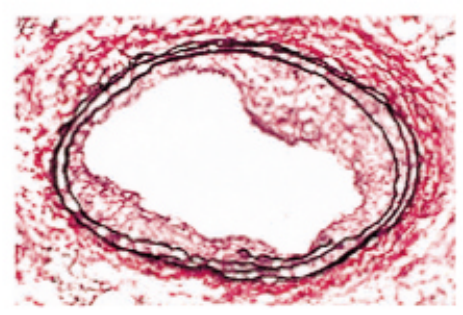

Figure 3

Medial thickening. (a) Representative sections of carotid arteries stained with Movat's pentachrome showing medial thickening in the nontransgenic injured compared with the sham or elafin transgenic-injured and sham vessels. (b) Graphs depict mean \pm SEM at day 10 after carotid arterial injury in elafin-transgenic $(n=9)$ and nontransgenic $(n=6)$ littermates. Medial thickening is quantified by the area between the innerand outermost elastic laminae. ${ }^{A} P=0.0004$ versus nontransgenic sham arteries. ${ }^{\mathrm{B}} P=0.036$ versus elafin transgenic-injured arteries. (c) Cell numbers calculated by counting nuclei in the intima + media. ${ }^{C} P=0.0001$ versus nontransgenic sham. ${ }^{D} P=0.0001$ versus elafin transgenic-injured arteries. (d) Neointima formation observed in the Movat's pentachrome stained section of an injured artery of one of the nontransgenic mice.
In the absence of documented inflammatory cell infiltration at 6 hours after endothelial denudation, the SMCs could be the source of elastase activity. Although we cannot exclude elastase production by adventitial cells, this is less likely, as the sham vessels were injured from the adventitial side. Moreover, we have previously described a serine elastase in SMCs that is induced by serum or endothelial factors through a MAP kinase pathway signaling increased nuclear expression and DNA binding of the transcription factor $\operatorname{AML} 1(7,16,17)$.

Elastase-induced degradation of extracellular matrices liberates growth factors such as FGF-2 (7) and PDGF (8) from vascular smooth muscle and endothelial cells. Inhibition of FGF-2 by anti-FGF- 2 antibodies reduces the medial thickening after carotid artery injury in mice (22). A synthetic thrombin inhibitor also prevents neointimal formation in the rat carotid injury by suppressing the release of PDGF, a potent chemoattractant as well as a growth factor (23).

Serine elastases regulate SMC migration and inflammatory cell infiltration of blood vessels by inducing the synthesis of platelet-activating factor (24) and generating proteolyzed fragments of laminin (25), elastin and fibronectin, which are chemoattractants. The process of migration of inflammatory cells may, therefore, be impeded by inhibition of elastases. This is supported by our previous observations showing reduced neutrophil infiltration in the myocardium after encephalomyocarditis virus infection in elafin-transgenic mice (12) and when a synthetic serine elastase inhibitor was administered (3). Gene transfer of elafin can also repress infiltration of neutrophils and macrophages in vein grafts (26). The initial blockade of inflammatory cell invasion by elafin can prevent amplification of the proteolytic cascade induced by invading neutrophils and macrophages that produce other serine proteinases, cathepsins, and matrix metalloproteinases (MMPs) $(27,28)$.

In the present study, loss of $\alpha$-actin expression in the nontransgenic mice at day 10 after injury was associated with increased cellularity in the media as well as intima, where migration of $\alpha$-actin-negative as well as -positive cells were observed. Loss of $\alpha$-actin has been described in balloon injury of carotid arteries $(18,20$, 29 ), coronary angioplasty (19), and post-transplant arteriosclerosis (30). This loss of $\alpha$-actin has been attributed to altered phenotype (29) and to proliferation of SMCs $(18,20)$. Exposure of arterial SMCs to PDGF and EGF also results in downregulation of $\alpha$ actin (31). In elafin-transgenic mice, preservation of actin expression could be due to the suppression of growth factor release and mitogenic stimulation of SMCs. Also, preservation of the integrity of the extracellular matrix may prevent dedifferentiation of SMCs as observed in carcinoma cells (32).

Loss of $\alpha$-actin in nontransgenic mice was associated with an increase in Mac-3 expression. While this 
a

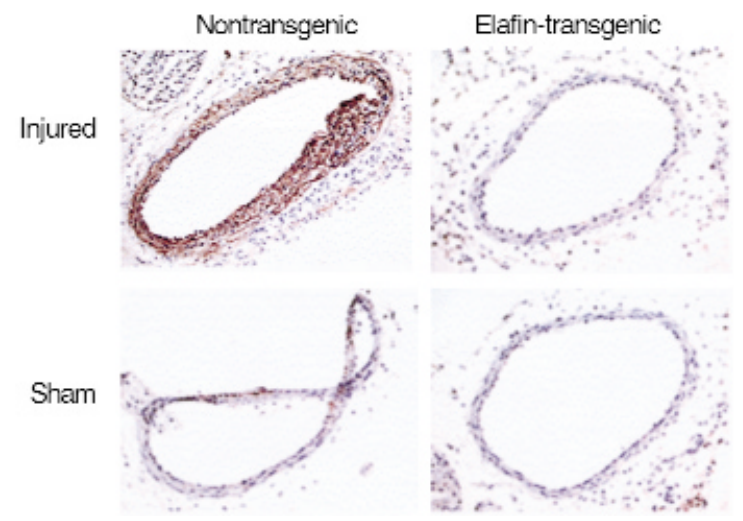

b

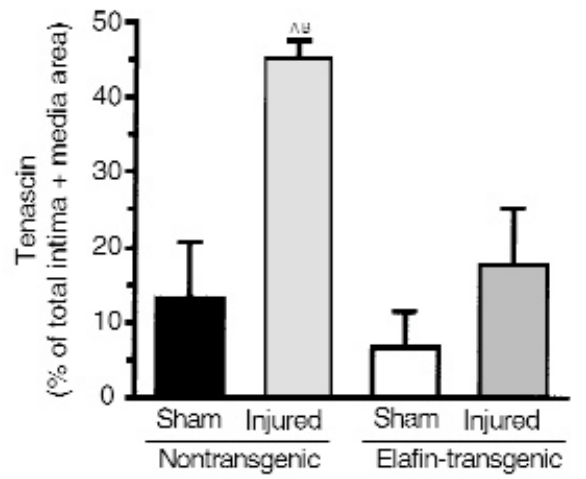

\section{Figure 4}

Tenascin protein expression. (a) Representative frozen sections of carotid arteries harvested at 10 days and showing increased immunostaining in nontransgenic versus elafin-transgenic mice throughout the thickened media of the injured artery. (b) Tenascin calculated as a percent of total intima plus media area from six nontransgenic littermates and nine elafin-transgenic mice. Bars represent mean \pm SEM. ${ }^{A} P=0.003$ versus nontransgenic sham. ${ }^{B} P=0.005$ versus elafin transgenic-injured arteries.

is attributable to infiltration of Mac-3-positive macrophages, it is also conceivable that SMC dedifferentiation is associated with Mac-3 expression as speculated earlier in transplanted carotid arteries in mice (30). Mac-3 antigen is also expressed by bone marrow cells (21) that have been observed at sites of skeletal muscle damage (33). Decreased medial accumulation of leukocytes in Mac- $1^{-/-}$mice also reduces neointimal thickening after carotid endothelial denudation (34). Ly-6G is a specific marker for neutrophils (15) and suggests that at least some of the increased cellularity is due to these inflammatory cells. Increased elastase activity seen at 7 days after endothelial injury in the CD1 mice likely results from these infiltrating inflammatory cells. Reduced inflammatory cell infiltration in the injured arteries of elafin-transgenic mice would be expected to result in suppression of serine elastase activity.
Both elastases and MMPs can upregulate the extracellular matrix protein tenascin that has been implicated in SMC proliferation after balloon carotid injuries (29) and progressive thickening and neointima formation in pulmonary arteries (35). In our vascular SMC and organ culture studies, we have observed (36) that heightened activity of elastases or MMPs leads to the unmasking of cryptic RGD (arginine-glycine-aspartic acid) sites on proteolyzed collagen that preferentially bind the $\beta 3$ integrin and induce MAP kinase-dependent transcription of the tenascin gene. When increased tenascin is produced, it codistributes with proteolyzed collagen in the matrix, resulting in the ligation and clustering of its cognate ligand, the $\beta 3$ integrin. This induces cell-shape changes associated with focal adhesion contacts and results in clustering of growth factor receptors, which are rapidly phosphorylated upon ligation, thus a

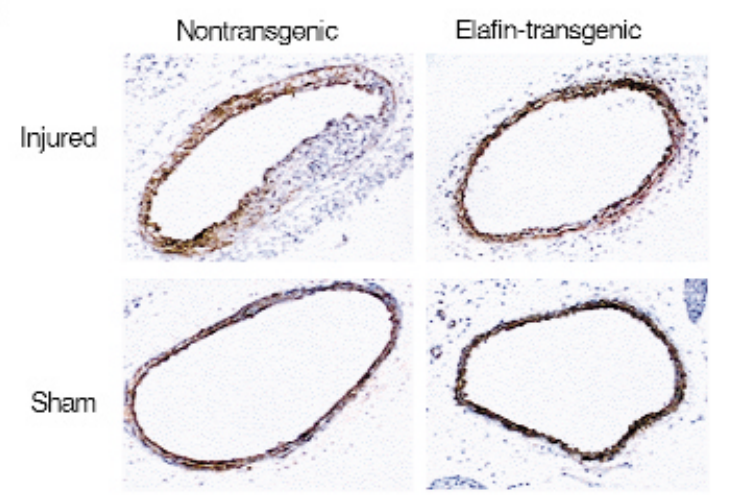

b

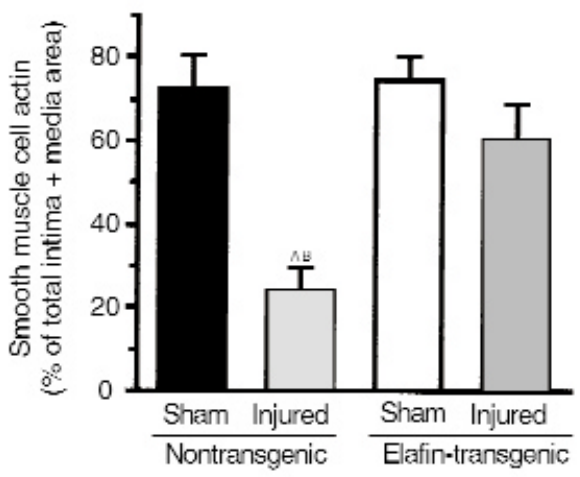

Figure 5

$\alpha$-Actin expression. (a) Representative frozen sections of injured and sham-operated carotid arteries harvested at 10 days and showing decreased immunostaining especially in the thickest part of the vessel wall in the nontransgenic-injured artery. (b) Area immunostained for actin was calculated as a percent of total intima plus media area. Bars represent mean \pm SEM from six nontransgenic littermates and nine elafin-transgenic mice. ${ }^{A} P=0.0002$ versus nontransgenic sham. ${ }^{B} P=0.0015$ versus elafin transgenic-injured arteries. 


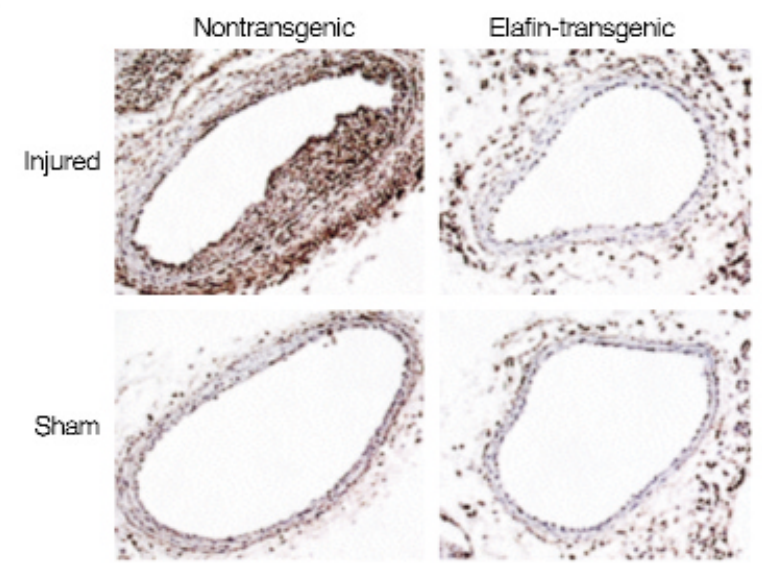

C

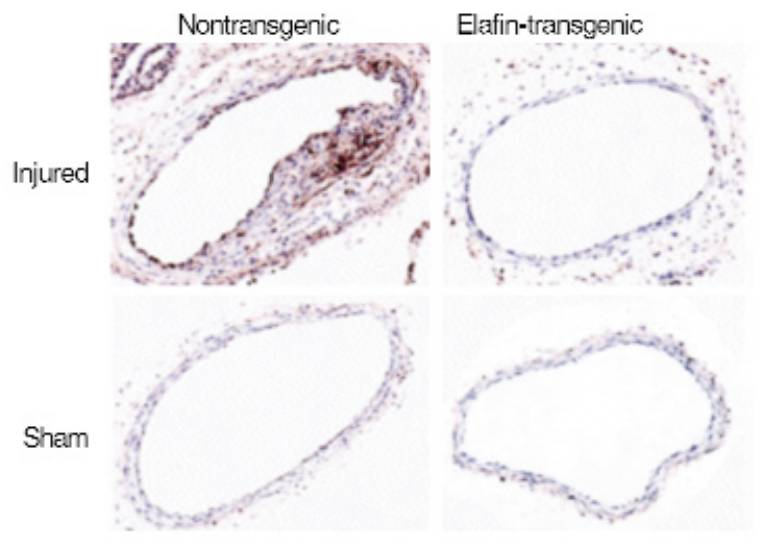

b

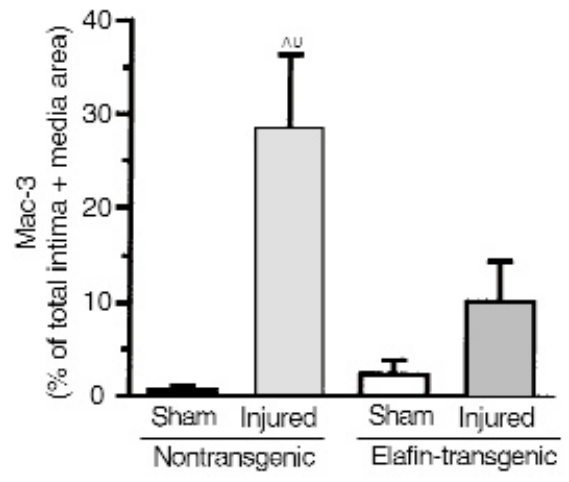

d

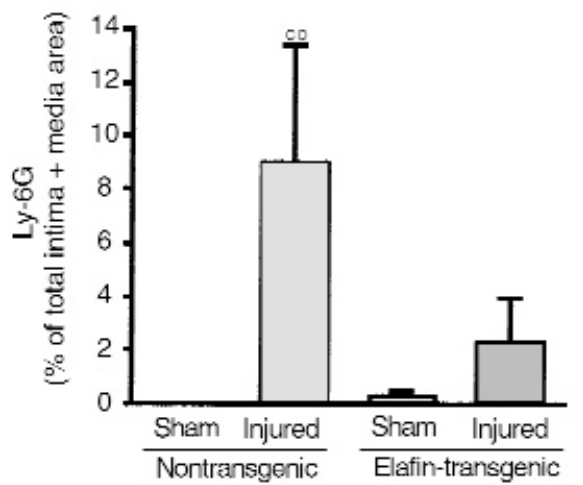

\section{Figure 6}

Mac-3 and Ly-6G expression. (a) Representative frozen sections from injured and sham-operated carotid arteries from nontransgenic and elafin-transgenic mice harvested at 10 days showing increased immunostaining for Mac-3 antigen in the injured vessel from the nontransgenic mouse. (b) Area with Mac-3 expression was quantified. Bars represent mean \pm SEM from six nontransgenic and nine elafin-transgenic littermates. ${ }^{A} P=0.0003$ versus nontransgenic sham. ${ }^{B} P=0.006$ versus elafin transgenic-injured arteries. (c) Representative frozen sections from injured and sham-operated carotid arteries at 10 days show increased immunostaining for a neutrophil marker Ly-6G in the nontransgenic mouse. (d) Area containing the Ly-6G staining was quantified. Bars represent mean \pm SEM from nine elafin-transgenic and six nontransgenic littermates. ${ }^{C} P=0.01$ versus nontransgenic sham. ${ }^{D} P=0.032$ versus elafin transgenic-injured arteries.

explaining how increased tenascin expression can amplify the proliferative response to growth factors. Induction of other proteinases such as MMPs and plasminogen activator have been reported after vascular injuries (37-41) and could have biologic effects similar to elastase. However, thickening of the vessel wall was not completely reduced after arterial injury when MMP inhibitors were used $(37,40,41)$ or in plasminogen knockout mice (42). Although elafin is highly selective in directly inhibiting serine elastases (14), it could also inhibit the elastase-dependent conversion of MMPs from a pro- to an active form $(4,5)$ or the inactivation of inhibitors of MMPs such as TIMP-1 by serine elastases (6). Serine elastases have also been implicated in the conversion of plasmin from plasminogen (43). Studies addressing the temporal upregulation of MMPs by others (38, 40, 41) suggest that they are induced several days after the early induction of serine elastase activity described here.

One of the most intriguing aspects of this study was positive remodeling in both the nontransgenic and elafin-transgenic injured arteries, at least as judged by an increased vessel size. This feature appears to be unrelated to the early induction of serine elastase activity. Thus, administration of elastase inhibitors might be expected to preserve positive remodeling after injury while protecting against thickening of the media and development of a neointima.

\section{Acknowledgments}

Supported by Heart and Stroke Foundation of Canada Grant HSFO 3170. M. Rabinovitch is a Research Endowed Chair of the Heart and Stroke Foundation of Canada and Distinguished Scientist of the Medical Research Council of Canada, and M. Husain is a Clini- 
cian Scientist of the Medical Research Council of Canada. The authors thank J. Jowlabar, J. Matthews, and J. Edwards for secretarial assistance.

1. Ye, C.L., and Rabinovitch, M. 1991. Inhibition of elastolysis by SC-37698 reduces development and progression of monocrotaline pulmonary hypertension. Am. J. Physiol. 261:H1255-H1267.

2. Cowan, B., et al. 1996. Elafin, a serine elastase inhibitor, attenuates postcardiac transplant coronary arteriopathy and reduces myocardial necrosis in rabbits after heterotopic cardiac transplantation. J. Clin. Invest. 97:2452-2468.

3. Lee, J.K., et al. 1998. A serine elastase inhibitor reduces inflammation and fibrosis and preserves cardiac function after experimentally-induced murine myocarditis. Nat. Med. 4:1383-1391.

4. Ferry, G., et al. 1997. Activation of MMP-9 by neutrophil elastase in an in vivo model of acute lung injury. FEBS Lett. 402:111-115.

5. Nagase, H., Enghild, J.J., Suzuki, K., and Salvesen, G. 1990. Stepwise activation mechanisms of the precursor of matrix metalloproteinase 3 (stromelysin) by proteinases and (4-aminophenyl)mercuric acetate. Biochemistry. 29:5783-5789.

6. Itoh, Y., and Nagase, H. 1995. Preferential inactivation of tissue inhibitor of metalloproteinase- 1 that is bound to the precursor of matrix metalloproteinase 9 (progelatinase B) by human neutrophil elastase. J. Biol. Chem. 270:16518-16521.

7. Thompson, K., and Rabinovitch, M. 1996. Exogenous leukocyte and endogenous elastases can mediate mitogenic activity in pulmonary artery smooth muscle cells by release of extracellular-matrix bound basic fibroblast growth factor. J. Cell. Physiol. 166:495-505.

8. Totani, L., Cumashi, A., Piccoli, A., and Lorenzet, R. 1998. Polymorphonuclear leukocytes induce PDGF release from IL-1beta-treated endothelial cells: role of adhesion molecules and serine proteases. Arterioscler. Thromb. Vasc. Biol. 18:1534-1540.

9. Wakasugi, K., and Schimmel, P. 1999. Two distinct cytokines released from a human aminoacyl-tRNA synthetase. Science. 284:147-151.

10. Boudreau, N., Turley, E., and Rabinovitch, M. 1991. Fibronectin, hyaluronan, and a hyaluronan binding protein contribute to increased ductus arteriosus smooth muscle cell migration. Dev. Biol. 143:235-247.

11. Cowan, K.N., Jones, P.L., and Rabinovitch, M. 2000. Elastase and matrix metalloproteinase inhibitors induce regression, and tenascin-C antisense prevents progression, of vascular disease. J. Clin. Invest. 105:21-34.

12. Zaidi, S.H., et al. 1999. Targeted overexpression of elafin protects mice against cardiac dysfunction and mortality following viral myocarditis. $J$. Clin. Invest. 103:1211-1219.

13. Lindner, V., Fingerle, J., and Reidy, M.A. 1993. Mouse model of arterial injury. Circ. Res. 73:792-796.

14. Sallenave, J.M., and Silva, A. 1993. Characterization and gene sequence of the precursor of elafin, an elastase-specific inhibitor in bronchial secretions. Am. J. Respir. Cell Mol. Biol. 8:439-445.

15. Hestdal, K., et al. 1991. Characterization and regulation of RB6-8C5 antigen expression on murine bone marrow cells. J. Immunol. 147:22-28.

16. Thompson, K., Kobayashi, J., Childs, T., Wigle, D., and Rabinovitch, M. 1998. Endothelial and serum factors which include apolipoprotein A1 tether elastin to smooth muscle cells inducing serine elastase activity via tyrosine kinase-mediated transcription and translation. J. Cell. Physiol. 174:78-89.

17. Wigle, D.A., et al. 1998. AML1-like transcription factor induces serine elastase activity in ovine pulmonary artery smooth muscle cells. Circ. Res. 83:252-263.

18. London, S.M., and Mayberg, M.R. 1994. Kinetics of bromodeoxyuridine uptake by smooth muscle cells after arterial injury. J. Vasc. Res. 31:247-255.

19. Ueda, M., Becker, A.E., Naruko, T., and Kojima, A. 1995. Smooth muscle cell de-differentiation is a fundamental change preceding wound healing after percutaneous transluminal coronary angioplasty in humans. Coron. Artery Dis. 1:71-81.

20. Thyberg, J., Blomgren, K., Roy, J., Tran, P.K., and Hedin, U. 1997. Phenotypic modulation of smooth muscle cells after arterial injury is associated with changes in the distribution of laminin and fibronectin. J. His- tochem. Cytochem. 45:837-846.

21. Ho, M.K., and Springer, T.A. 1983. Tissue distribution, structural characterization, and biosynthesis of Mac-3, a macrophage surface glycoprotein exhibiting molecular weight heterogeneity. J. Biol. Chem. 258:636-642.

22. Bryant, S.R., Bjercke, R.J., Erichsen, D.A., Rege, A., and Lindner, V. 1999. Vascular remodeling in response to altered blood flow is mediated by fibroblast growth factor-2. Circ. Res. 84:323-328.

23. Sawada, M., et al. 1999. Prevention of neointimal formation by a serine protease inhibitor, FUT-175, after carotid balloon injury in rats. Stroke. 30:644-650.

24. Bussolino, F., and Camussi, G. 1995. Platelet-activating factor produced by endothelial cells. A molecule with autocrine and paracrine properties. Eur. J. Biochem. 229:327-337.

25. Steadman, R., et al. 1993. Laminin cleavage by activated human neutrophils yields proteolytic fragments with selective migratory properties. J. Leukoc. Biol. 53:354-365.

26. O'Blenes, S.B., et al. 2000. Gene transfer of the serine elastase inhibitor elafin protects against vein graft degeneration. Circulation. In press.

27. Caughey, G.H. 1994. Serine proteinases of mast cell and leukocyte granules. A league of their own. Am. J. Respir. Crit. Care Med. 150(Suppl.):S138-S142.

28. Shapiro, S.D. 1994. Elastolytic metalloproteinases produced by human mononuclear phagocytes. Potential roles in destructive lung disease. Am. J. Respir. Crit. Care Med. 150(Suppl.):S160-S164.

29. Hedin, U., Holm, J., and Hansson, G.K. 1991. Induction of tenascin in rat arterial injury. Relationship to altered smooth muscle cell phenotype. Am. J. Pathol. 139:649-656.

30. Moons, L., et al. 1998. Reduced transplant arteriosclerosis in plasminogen-deficient mice. J. Clin. Invest. 102:1788-1797.

31. Bjorkerud, S. 1991. Effects of transforming growth factor-beta 1 on human arterial smooth muscle cells in vitro. Arterioscler. Thromb. 11:892-902.

32. Bissell, M.J., et al. 1999. Tissue structure, nuclear organization, and gene expression in normal and malignant breast. Cancer Res. 59(Suppl. 7):1757S-1763S.

33. Ferrari, G., et al. 1998. Muscle regeneration by bone marrow-derived myogenic progenitors. Science. 279:1528-1530.

34. Simon, D.I., et al. 2000. Decreased neointimal formation in Mac-1(-/-) mice reveals a role for inflammation in vascular repair after angioplasty. J. Clin. Invest. 105:293-300.

35. Jones, P.L., and Rabinovitch, M. 1996. Tenascin-C is induced with progressive pulmonary vascular disease in rats and is functionally related to increased smooth muscle cell proliferation. Circ. Res. 79:1131-1142.

36. Jones, P.L., Jones, F.S., Zhou, B., and Rabinovitch, M. 1999. Induction of vascular smooth muscle cell tenascin-C gene expression by denatured type I collagen is dependent upon a beta 3 integrin-mediated mitogenactivated protein kinase pathway and a 122-base pair promoter element. J. Cell Sci. 112:435-445.

37. Prescott, M.F., et al. 1999. Effect of matrix metalloproteinase inhibition on progression of atherosclerosis and aneurysm in LDL receptor-deficient mice overexpressing MMP-3, MMP-12, and MMP-13 and on restenosis in rats after balloon injury. Ann. NY Acad. Sci. 878:179-190.

38. Lijnen, H.R., Silence, J., Van Hoef, B., and Collen, D. 1998. Stromelysin1 (MMP-3)-independent gelatinase expression and activation in mice. Blood. 91:2045-2053.

39. Carmeliet, P., et al. 1997. Urokinase but not tissue plasminogen activator mediates arterial neointima formation in mice. Circ. Res. 81:829-839.

40. Strauss, B.H., et al. 1996. In vivo collagen turnover following experimental balloon angioplasty injury and the role of matrix metalloproteinases. Circ. Res. 79:541-550.

41. Bendeck, M.P., Irvin, C., and Reidy, M.A. 1996. Inhibition of matrix metalloproteinase activity inhibits smooth muscle cell migration but not neointimal thickening after arterial injury. Circ. Res. 78:38-43.

42. Carmeliet, P., Moons, L., Ploplis, V., Plow, E., and Collen, D. 1997. Impaired arterial neointima formation in mice with disruption of the plasminogen gene. J. Clin. Invest. 99:200-208.

43. Machovich, R., Himer, A., and Owen, W.G. 1990. Neutrophil proteases in plasminogen activation. Blood Coagul. Fibrinolysis. 1:273-277. 